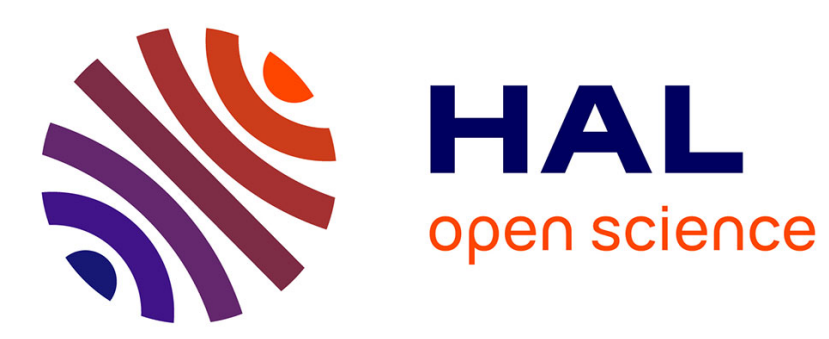

\title{
Accélération de particules dans le milieu interplanétaire
}

\author{
J.J. Engelmann
}

\section{To cite this version:}

J.J. Engelmann. Accélération de particules dans le milieu interplanétaire. Revue de Physique Appliquée, 1988, 23 (9), pp.1535-1543. 10.1051/rphysap:019880023090153500 . jpa-00245982

\section{HAL Id: jpa-00245982 https://hal.science/jpa-00245982}

Submitted on 1 Jan 1988

HAL is a multi-disciplinary open access archive for the deposit and dissemination of scientific research documents, whether they are published or not. The documents may come from teaching and research institutions in France or abroad, or from public or private research centers.
L'archive ouverte pluridisciplinaire HAL, est destinée au dépôt et à la diffusion de documents scientifiques de niveau recherche, publiés ou non, émanant des établissements d'enseignement et de recherche français ou étrangers, des laboratoires publics ou privés. 


\title{
Accélération de particules dans le milieu interplanétaire
}

\author{
J. J. Engelmann \\ Service d'Astrophysique, CEN Saclay, France
}

(Reçu le 15 octobre 1987, accepté le 17 mai 1988)

\begin{abstract}
Résumé. - Le vent solaire présente des caractéristiques variables, fonction de la configuration des courants de vent de grande vitesse qui y sont présents. Certains de ces courants, dus à des bouffées de matière coronale éjectées lors d'éruptions solaires, sont transitoires, d'autres, qui ont pour origine des « trous coronaux », peuvent durer une fraction notable de rotation solaire. Par interaction avec le vent calme, ces courants rapides génèrent, dans le premier cas, une onde de choc qui se propage dans le milieu interplanétaire, dans le second cas une paire d'ondes de choc, l'une vers l'extérieur, l'autre vers l'intérieur de la cavité solaire, qui délimitent une zone d'interaction en corotation avec le soleil. Dans les deux cas, on observe des flux d'ions énergiques $(\sim \mathrm{MeV})$ en association avec les ondes de choc ; ce sont respectivement « les particules énergiques d'orage » et «les événements particulaires en corotation». Deux mécanismes d'accélération sont invoqués pour rendre compte de ces accroissements de flux : 1) Le mécanisme de Fermi de $1^{\text {er }}$ ordre, dans lequel les particules gagnent de l'énergie par diffusion entre des irrégularités magnétiques entraînées par le vent solaire de part et d'autre de l'onde de choc. 2) Le mécanisme de dérive dans le choc, dans lequel les particules gagnent de l'énergie par dérive dans le champ électrique $\mathbf{v} \wedge \mathbf{B}$ associé au front d'onde. Ce dernier mécanisme est surtout efficace pour des ondes de choc quasi perpendiculaires. Il rend bien compte des événements brefs, de quelques minutes de durée. La composition et le spectre des ions accélérés suggèrent qu'ils ont probablement pour origine la queue de la distribution suprathermique du vent solaire.
\end{abstract}

\begin{abstract}
Variations in solar wind properties are dominated by a number of high speed streams. Some of them, due to bubbles of coronal matter ejected during solar flares, are transient, others, originating from coronal holes last for a significant fraction of a solar rotation. By interacting with the quiet wind, these fast streams give rise in the first case to a travelling shock wave, in the second case to a pair of forward and backward shock waves, by which the interaction region, corotating with the sun, is bounded. In both cases, high fluxes of energetic ions $(\sim \mathrm{MeV})$ are observed in the vicinity of the shock : these are « the energetic storm particles » and « the corotating particle events » respectively. Two acceleration mechanisms are invoked to account for these ion flux increases : 1) The first order Fermi process, whereby particles increase their energy by compression between converging magnetic scattering centers, located upstream and downstream of the shock. 2) The shock drift mechanism, whereby particles gain energy by drifting through the $\mathbf{v} \times \mathbf{B}$ electric field associated with the shock front; this mechanism is most effective with quasi-perpendicular shocks, and accounts well for shock spike events. The composition and the spectrum of the accelerated ions suggest that they probably originate from the suprathermal tail of the solar wind distribution.
\end{abstract}

\section{Introduction.}

Une des plus importantes découvertes de ces quinze dernières années d'exploration spatiale est l'étroite association entre les ondes de choc et les accroissements de flux de particules de grande énergie (jusqu'à quelques dizaines de $\mathrm{MeV}$ ) dans l'espace interplanétaire. Ce dernier est balayé en permanence par le vent solaire, qui n'est autre que l'extension de la couronne solaire, dont la température est de l'ordre de 2 millions de degrés. La couronne consti- tue la partie la plus externe de l'atmosphère solaire. Elle n'est séparée de la photosphère autour de $6000^{\circ}$ que par la chromosphère, mince couche $(\sim 2000 \mathrm{~km})$ où la température remonte lentement d'un minimum vers $4500^{\circ}$ jusqu'à $10000 \mathrm{~K}$. Entre la chromosphère et la couronne existe une très fine région de transition dans laquelle la température grimpe brutalement (Fig. 1) [1].

Du fait de la haute température de la couronne, l'énergie cinétique des électrons coronaux est supérieure à celle de leur liaison gravitationnelle avec le 


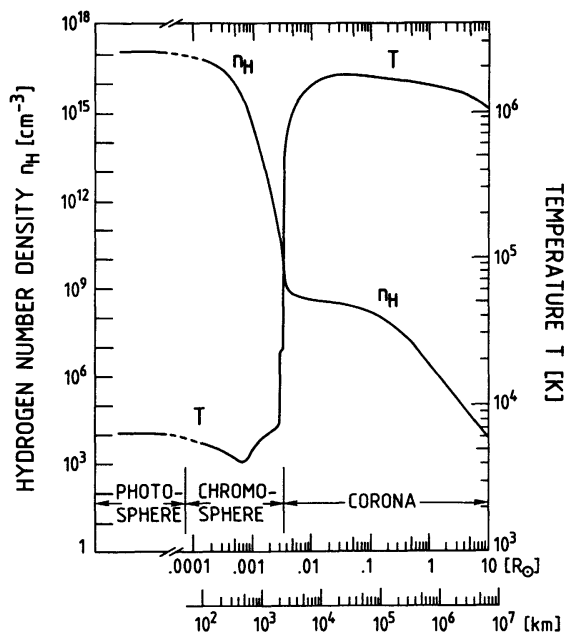

HEIGHT ABOVE PHOTOSPHERE

Fig. 1. - Représentation schématique de l'atmosphère solaire externe, définissant la chromosphère et la couronne, qui sont séparées par une région de transition très fine dans laquelle la température monte très rapidement. La densité et la température de l'hydrogène sont portées en fonction de la hauteur au-dessus de la photosphère (d'après [1]).

[Idealized sketch of the outer solar atmosphere, defining the chromosphere and the corona, which are separated by a very thin transition region in which the temperature rises extremely abruptly. Hydrogen density and temperature are plotted against height above photosphere (from [1]).]

soleil. Ils s 'échappent dans l'espace et créent dans la couronne un déséquilibre de charge électrique qui, à son tour, provoque l'échappement des protons dans le milieu interplanétaire. La différence de pression entre la basse couronne et le milieu interplanétaire est telle que le vent solaire atteint une vitesse supérieure à celle des perturbations de densité qui peuvent s'y propager; il s'écoule à une vitesse supersonique, de l'ordre de $400 \mathrm{~km} / \mathrm{s}$ en moyenne $(\sim 1 \mathrm{keV})$. La densité des ions, en majorité des protons, qui était voisine de $10{ }^{10} \mathrm{~cm}^{-3}$ dans la basse couronne, au voisinage de la zone de transition, décroît en gros suivant l'inverse carré de la distance au soleil et n'est plus en moyenne que de $6 \mathrm{~cm}^{-3}$ à l'orbite de la terre (autrement dit à une distance d'une unité astronomique [u.a.] du soleil).

Par ailleurs, le soleil a une configuration magnétique complexe : un champ général dipolaire le long de l'axe de rotation (le soleil tourne sur lui-même en 27 jours autour d'un axe presque perpendiculaire au plan de l'écliptique) sur lequel se superposent de fortes irrégularités de champ, liées aux taches photosphériques. Dans les régions de plus faible champ magnétique, le vent solaire entraîne le champ magnétique, qui est gelé dans le gaz. Du fait de la rotation solaire, les particules du vent solaire qui ont quitté un point de la couronne à des instants

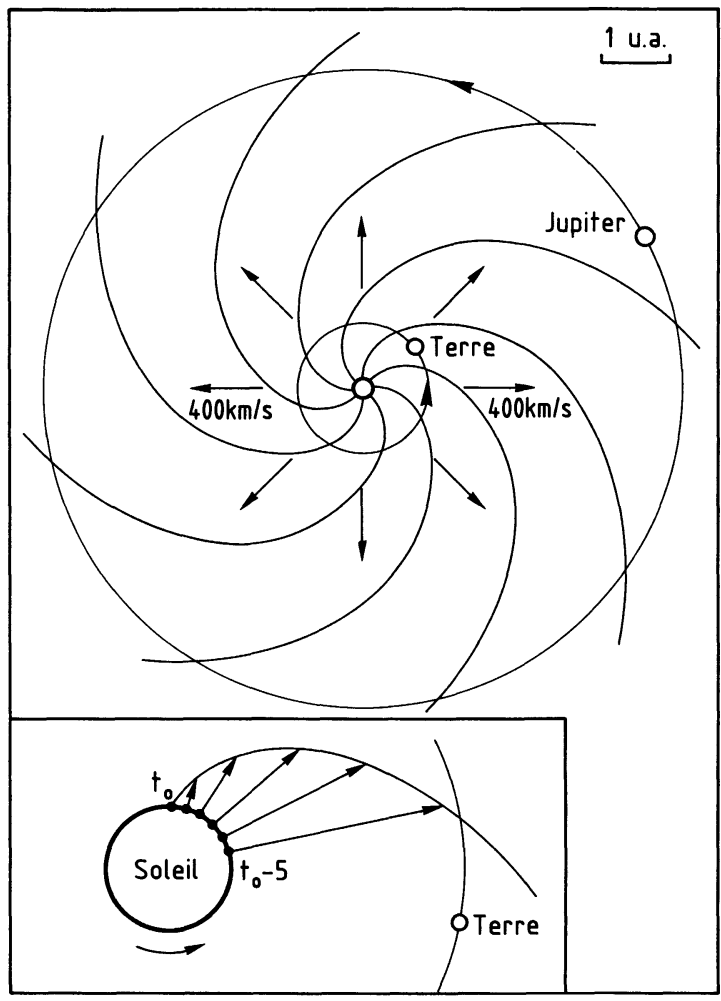

Fig. 2. - Structure du champ magnétique dans le plan de l'écliptique. Les particules du vent solaire qui ont quitté un point de la basse couronne à des instants successifs en entraînant le champ magnétique se trouvent à une distance d'autant plus grande du soleil qu'elles ont été émises plus tôt. Leur lieu géométrique est une spirale d'Archimède.

[Magnetic field structure in the ecliptic plane. Due to the combination of the radial flow of the solar wind and of the solar rotation, the magnetic plasma originating from a certain point of the lower corona is localized along an Archimedes spiral.]

successifs se retrouvent sur une spirale d'Archimède dans l'espace interplanétaire (Fig. 2) [2, 3, 4].

\section{Processus de formation d'une onde de choc dans l'espace interplanétaire.}

La vitesse du vent solaire n'est ni constante dans le temps, ni uniforme dans l'espace, du fait de l'évolution temporelle et spatiale de la configuration magnétique de la photosphère et de la couronne. Lors de l'interaction d'un courant de vent solaire rapide avec le vent calme, une onde de choc peut être générée. Cela se produit en particulier dans les deux cas suivants :

2.1. - Lors des éruptions solaires (« Solar Flares »), des bouffées de matière coronale sont éjectées et se propagent dans l'espace interplanétaire. Une onde de choc se forme alors à l'interface entre cette bouffée de plasma rapide et le vent solaire permanent. Un satellite, suivant sa position par rapport à 
l'éruption, peut observer le passage d'une onde de choc soit parallèle (dont la normale au front d'onde est parallèle au champ magnétique interplanétaire), soit oblique, soit perpendiculaire. Souvent, le satellite observe simultanément un accroissement de flux de particules énergiques (essentiellement des protons, d'énergie généralement inférieure à quelques $\mathrm{MeV}$ ) (Fig. 3). L'orientation de l'onde de choc par rapport au champ magnétique joue un rôle dans le mécanisme d'accélération, comme on le verra plus loin. Lorsque l'onde de choc arrive au voisinage de la terre, sur la magnétosphère, elle provoque un orage magnétique. Il y a donc une corrélation entre les particules accélérées dans l'espace interplanétaire et l'orage magnétique enregistré sur terre. Aussi appelle-t-on celles-ci particules énergiques d'orage («Energetic Storm particles »).

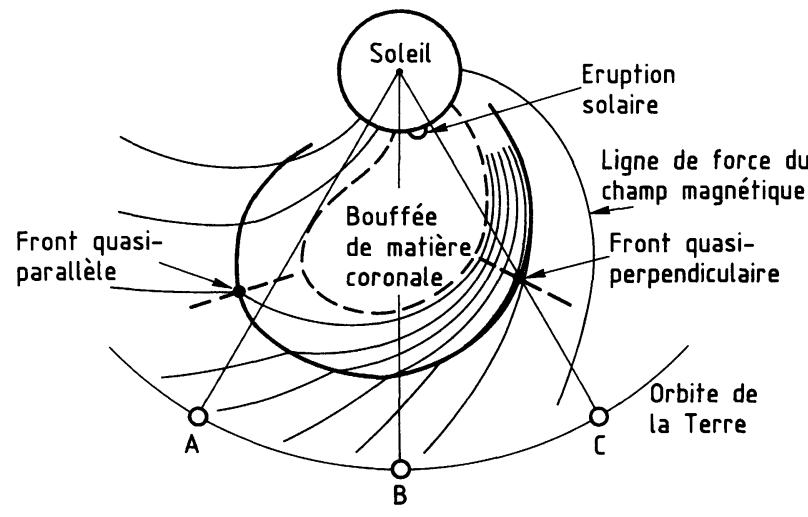

Fig. 3. - Onde de choc produite par une bouffée de matière coronale éjectée lors d'une éruption solaire. Suivant la position de la terre sur son orbite, un satellite proche-terrestre verra soit une onde de choc quasi parallèle (en A), soit oblique (en B), soit quasi perpendiculaire (en C). Des particules accélérées sont observées au voisinage du front d'onde [21].

[Shock wave caused by a bubble of coronal matter ejected during a solar flare. Depending on the Earth's position on its orbit, a near-Earth satellite will see either a quasiparallel (A), or an oblique (B), or a quasi-perpendicular shock wave (C). Accelerated particles are observed in the shock's vicinity [21].]

2.2. - Des courants de vent solaire rapide ont pu être associés à des structures dans la couronne : les trous coronaux, qui rayonnent particulièrement peu dans les domaines $\mathrm{X}$, UV et radiométrique. Ces structures sont des régions de faible densité et température, qui apparaissent dans des zones de champ magnétique faible et unipolaire, donc dans lesquelles les lignes de force du champ sont ouvertes et divergentes. Le vent solaire rapide qui en sort entre en interaction avec le vent calme provenant des régions de longitude voisine; une double onde de choc se forme au-delà de 1,5 u.a. (où les lignes de champ deviennent de plus en plus tangentielles (Figs. 2 et 4)) : l'une vers l'extérieur de la cavité solaire, dans laquelle le vent solaire lent est accéléré brutalement, l'autre, vers l'intérieur, dans laquelle le vent solaire rapide est décéléré (Fig. 4). Comme la configuration des trous coronaux peut rester stationnaire sur plusieurs rotations solaires, la configuration de la région d'interaction peut, elle aussi, rester stationnaire dans un système de référence en corotation avec le soleil. Des particules sont accélérées en permanence par ces ondes de choc et un observateur lié à la terre verra des accroissements de flux de particules énergiques («Corotating Ion Events ») avec une périodicité de 25 jours (période de rotation apparente du soleil, vue de la terre).

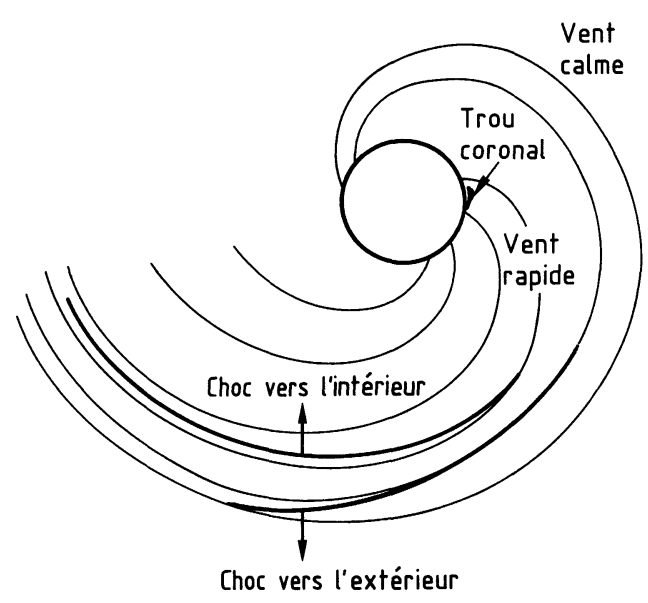

Fig. 4. - Ondes de choc produites par la rencontre d'un courant de vent rapide issu d'un trou coronal avec le vent calme (dans un système de référence en rotation avec le soleil). Au-delà de 1,5 u.a. deux ondes de choc se forment, l'une vers l'avant, par rapport au soleil, l'autre vers l'arrière ; elles délimitent la zone d'interaction en corotation, dans laquelle des particules sont accélérées [3].

[Interaction of a fast stream of solar wind, originating from a coronal hole, with the ambient solar wind, as viewed in a frame of reference rotating with the sun. Beyond 1,5 u.a., a pair of shock waves develop at the edges of the interaction region : one is a forward shock, propagating out of the sun, the other a reverse shock. High fluxes of energetic ions are observed in the vicinity of the interaction region [3].]

\section{Observations de particules accélérées dans l'espace interplanétaire.}

L'exploration des planètes géantes, de Jupiter à Neptune, a commencé dans les années 1970, grâce aux sondes de reconnaissance Pioneer 10 et 11, puis, à partir de 1977, grâce aux sondes plus sophistiquées, Voyager 1 et 2 . Toutes ces sondes étaient équipées pour mesurer les caractéristiques du champ magnétique interplanétaire, du vent solaire et des flux de particules énergiques $(\sim \mathrm{MeV})$ chargées. Ainsi, au 
cours de leur voyage interplanétaire, entre leurs rendez-vous avec les planètes, elles ont pu détecter des événements particulaires corrélés à des ondes de choc, et cela, à des distances du soleil allant de 1 à 30 u.a. A l'heure actuelle, les sondes Voyager poursuivent leur chemin dans l'espace et Voyager 2 arrivera au voisinage de Neptune à 30 u.a. en août 1989 , soit 12 ans après son lancement.

Grâce à ces sondes et aux satellites proche-terrestres (entre autres ISEE 3 «International Sun-Earth Explorer », lancé fin 1978), on a pu suivre l'évolution des ondes de choc durant leur propagation ainsi que celle des événements particulaires associés.

La fréquence des deux types d'accélération discutés au paragraphe 2 varie au cours du cycle de 11 ans de l'activité solaire. En période de maximum d'activité, de nombreuses «tâches solaires", régions actives de champ magnétique intense et turbulent, apparaissent sur la photosphère. C'est pourquoi les particules énergiques d'orage sont observées préférentiellement au voisinage du maximum d'activité solaire. Par contre, c'est lorsque le soleil est le plus calme que les trous coronaux polaires s'étendent vers de plus basses latitudes et donnent naissance à des courants de vent rapide au voisinage du plan de l'écliptique [5]. Les événements en corotation sont donc surtout observés au voisinage du minimum d'activité solaire.

3.1 EVÉNEMENTS PARTICULAIRES D'ORAGE. - Un exemple d'un tel événement, enregistré par Voyager 1 lorsqu'il était situé à 1,65 u.a., du soleil est présenté figure 5. On remarque que l'intensité maximale du flux de particules a lieu, pour toutes les énergies, au moment du passage du front de l'onde de choc, mais que la plus grosse partie de l'accroissement de flux a lieu après le passage du choc pour les basses énergies, mais avant son passage pour les hautes énergies [6].

De tels événements ont encore lieu à grande distance du soleil. C'est ainsi que Pioneer 10 a enregistré l'événement présenté figure 6 alors qu'il se trouvait à une distance de 28 u.a. du soleil. On remarque que l'onde de choc a accéléré non seulement des ions jusqu'à des énergies de quelques dizaines de $\mathrm{MeV}$, mais aussi des électrons jusqu'à des énergies relativistes, ce qui est un phénomène plus rare $[7,8]$.

Les accroissements de flux durent en général de quelques heures à quelques jours. Le spectre d'énergie des ions accélérés peut être bien représenté par une loi en puissance de l'énergie avec un indice spectral de 2 à $3[9,10]$.

3.2 EVÉNEMENTS RÉCURRENTS ASSOCIÉS AUX RÉGIONS D'INTERACTION EN COROTATION. - Un exemple d'un tel événement est représenté figure 7, où sont comparés les taux de comptage de protons

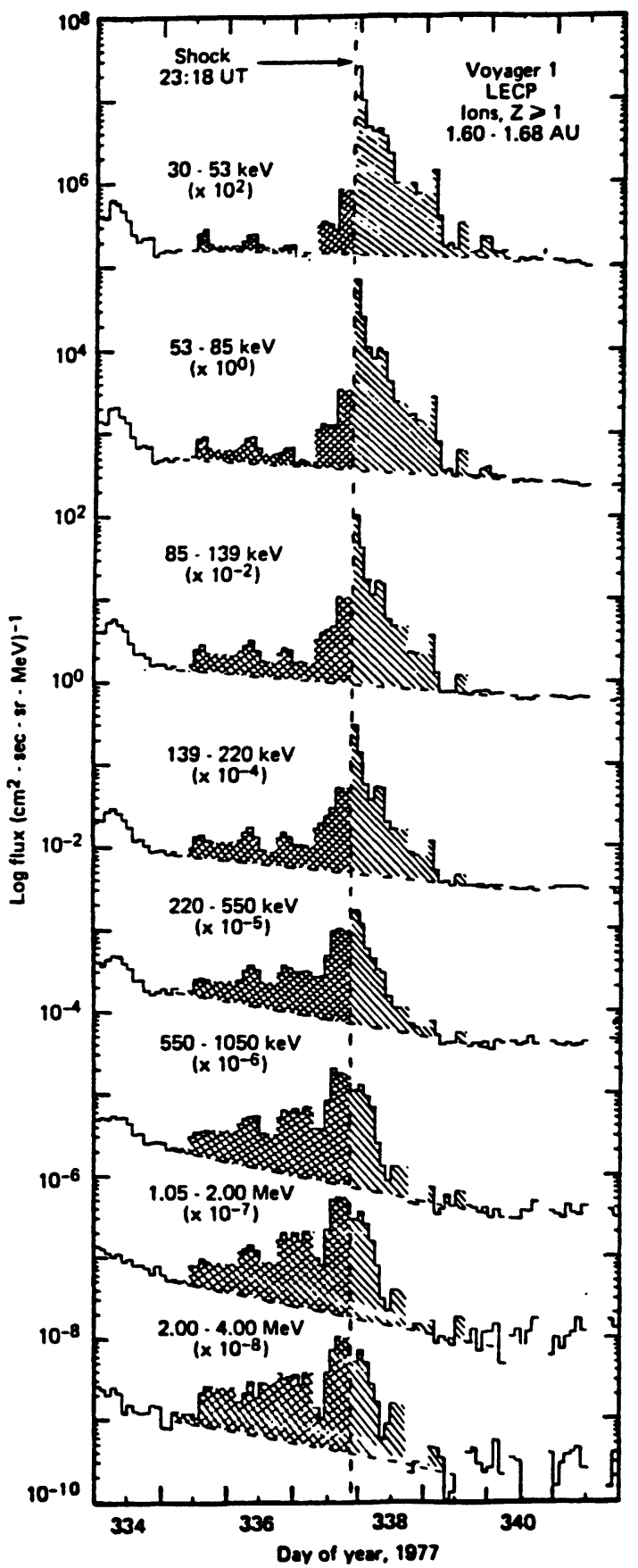

Fig. 5. - Evénement particulaire d'orage, enregistré par la sonde Voyager 1, lorsqu'elle se trouvait à environ 1,65 unité astronomique du soleil. Les accroissements de flux observés avant et après le passage de l'onde de choc ont été hachurés différemment pour faire ressortir la dépendance en énergie de la morphologie de l'accroissement de flux (d'après [6]).

[Energetic storm particle event observed by Voyager 1 at 1.65 u.a. from the sun. The pre-shock and post-shock particle intensities are shaded differently to emphasize the energy dependence of the enhancement morphologies (from [6]).]

enregistrés par trois détecteurs identiques placés à bord des satellites IMP 8, Pioneer 11 et Pioneer 10, 


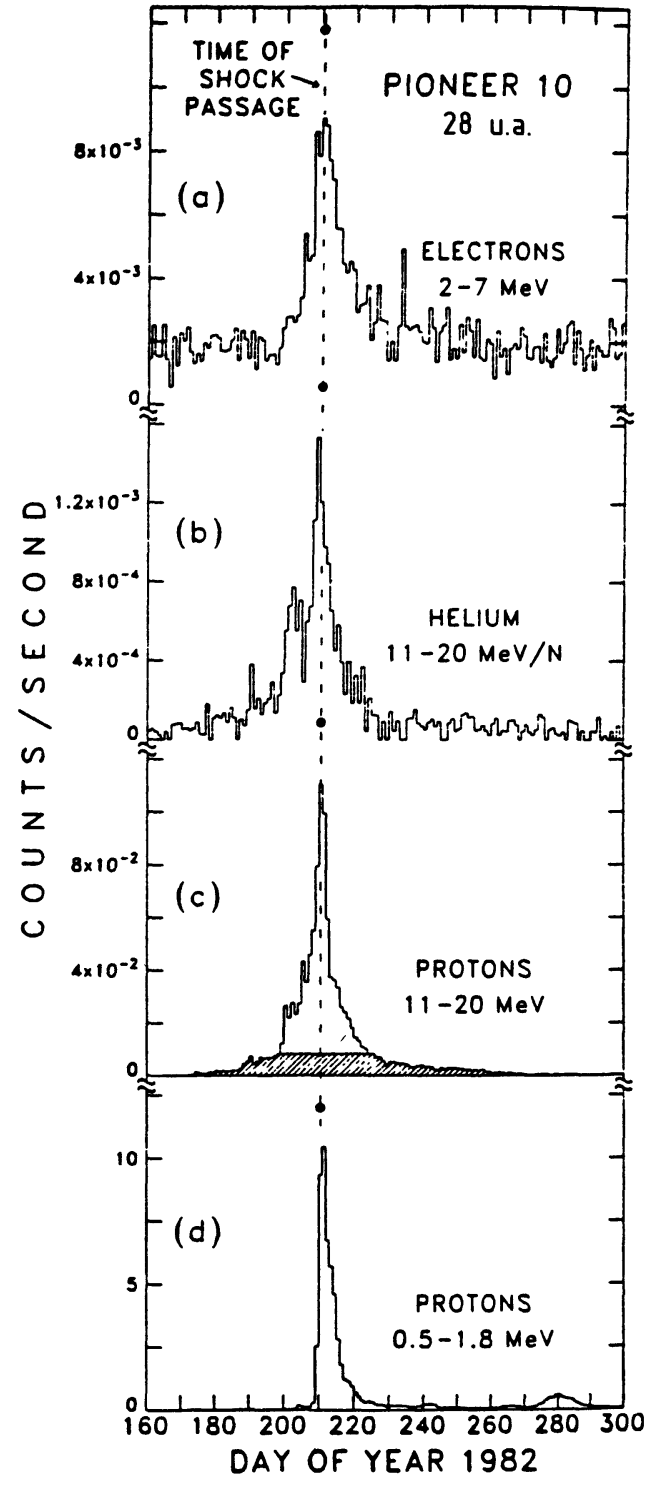

Fig. 6. - Evénement particulaire d'orage, enregistré par la sonde Pioneer 10 lorsqu'elle se trouvait à une distance de 28 u.a. du soleil. La zone hachurée en (c) représente la contribution probable des particules solaires au taux de comptage (d'après [7]).

[Energetic storm particle event observed by Pioneer 10 at 28 u.a. from the sun. Shading in (c) indicates the probable contribution of solar particles to the counting rate (from [7]).]

situés respectivement à environ $1,4,5$ et 5,8 u.a. du soleil [11]. On remarque que tous les 27 jours, à chaque passage de la région d'interaction, un double pic de protons est enregistré par Pioneer 10 et 11 ; ce double pic correspond au passage de la double onde de choc, directe et inverse, mentionnée ci-dessus $[12,13]$.

\section{Mécanismes d'accélération.}

Deux mécanismes sont invoqués pour rendre compte de ces divers types d'événements, et leur

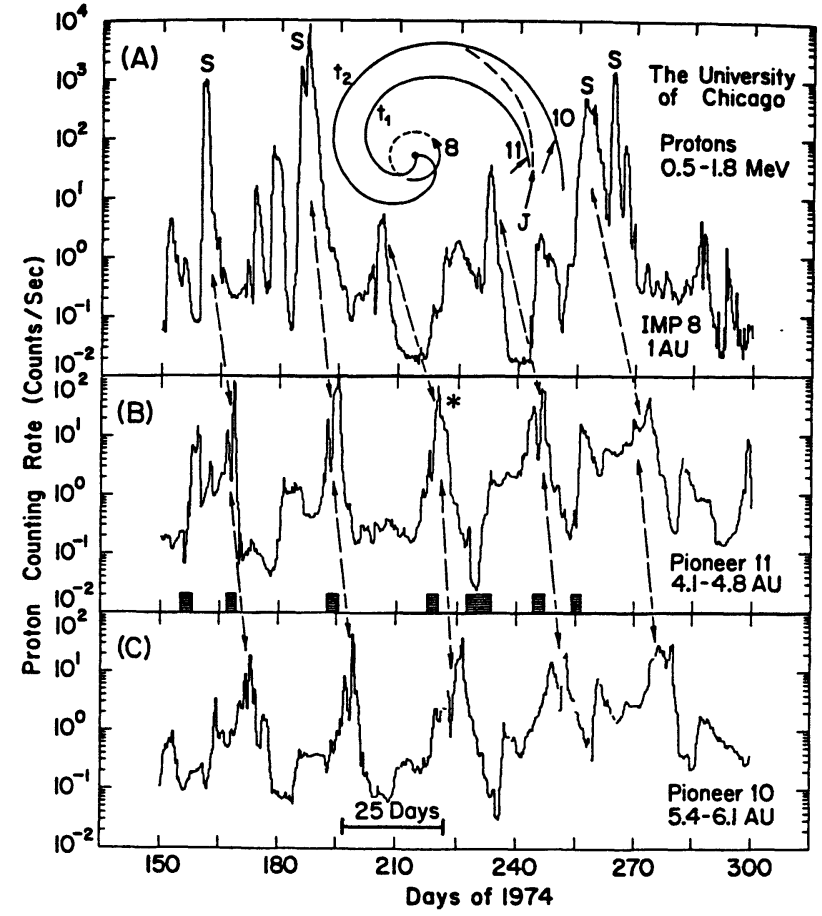

Fig. 7. - Evénements récurrents associés à une région d'interaction en corotation. Sur la figure sont comparés les flux de protons d'énergie $0,5-1,8 \mathrm{MeV}$, détectés par le satellite proche-terrestre IMP8 et par les sondes interplanétaires Pioneer 11 et Pioneer 10. Les zones hachurées horizontales représentent les passages des zones d'interaction en corotation. L'encart en haut de la figure montre les trajectoires de IMP8, Pioneer 11 et Pioneer 10 pendant la période considérée (d'après [11]).

[Recurrent particle event associated with a corotating interaction region(CIR). Proton counting rates from identical detectors on the near-Earth satellite IMP8 and on the interplanetary probes Pioneer 11 and Pioneer 10. The horizontally hatched areas correspond to CIR passages. The inset at top shows the trajectories of the spacecraft and Jupiter during this period of time (from [11]).]

importance relative est encore très controversée. L'un est le mécanisme de Fermi de $1^{\text {er }}$ ordre [14, 15], l'autre le mécanisme d'accélération par dérive dans le choc (Shock Drift Acceleration Mechanism) $[16,17,18]$.

4.1 MÉCAnismes DE FERMI DE $1^{\mathrm{er}}$ ORDRE. Lorsqu'il y a des irrégularités qui sont entraînées par le vent solaire de part et d'autre de l'onde de choc, des particules peuvent gagner de l'énergie en étant diffusées élastiquement entre ces irrégularités : il y a compression des particules entre les centres de diffusion puisque ceux qui s'approchent du choc en aval y arrivent plus vite que ceux qui s'en éloignent en amont. C'est un processus stochastique mais dans lequel une particule individuelle ne peut que gagner de l'énergie à chaque aller et retour à travers l'onde de choc (contrairement au mécanisme de Fermi de 
(a)

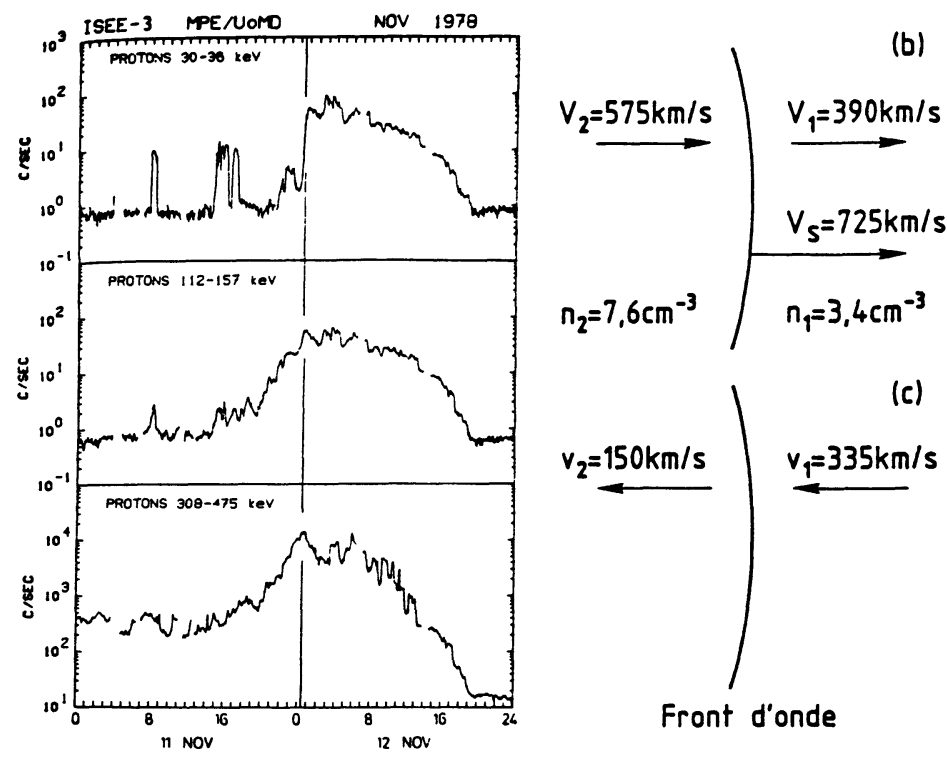

Fig. 8. - Evénement des 11-12 novembre 1978: un exemple d'accélération par une onde de choc quasi parallèle. a) Flux de protons enregistrés dans différentes gammes d'énergie par le satellite ISEE 3 (d'après [19]). b) Caractéristiques du vent solaire mesurées avant $\left(V_{1}, n_{1}\right)$ et après $\left(V_{2}, n_{2}\right)$ le passage de l'onde de choc. $V_{\mathrm{s}}:$ vitesse de l'onde de choc. c) Vitesse du vent solaire de part et d'autre de l'onde de choc dans un référentiel lié au front de l'onde.

[The 11-12 November 1978 event, an example of acceleration by a quasi-parallel shock wave. a) Proton fluxes measured in different energy intervals by the ISEE 3 satellite (from [19]). b) Solar wind characteristics measured before $\left(V_{1}, n_{1}\right)$ and after $\left(V_{2}, n_{2}\right)$ the shock wave passage. $V_{s}$ : shock wave velocity. c) Solar wind velocity on both sides of the shock wave in the shock front frame of reference.]

$2^{c}$ ordre dans lequel une particule peut tantôt gagner, tantôt perdre de l'énergie, mais avec un gain d'énergie global pour une population de particules). Ces mécanismes et leur intérêt général ont été discutés ici par O. Drury [32]. Pour une particule de moment $p$ et une onde de choc parallèle, le gain moyen par traversée est, dans le cas non relativiste :

$$
\langle\Delta p\rangle=\frac{2}{3} m\left(1-\frac{1}{r}\right) v_{2}
$$

où $m$ est la masse de la particule, $v_{2}$ la vitesse du vent solaire en amont de l'onde et $r$ est le rapport des densités en amont et en aval de l'onde. Lorsque l'équilibre est atteint, le flux différentiel en moment est de la forme [23] :

$$
\frac{\mathrm{d} \phi}{\mathrm{d} p}=p^{1-\frac{s}{2}} \quad \text { avec } \quad s=\frac{3 r}{r-1} .
$$

Un événement typique, observé par ISEE 3 est illustré dans la figure 8 . L'onde de choc à laquelle était associé l'accroissement de flux de protons était du type quasi parallèle [19]. Les caractéristiques du vent solaire de part et d'autre de l'onde de choc ont été portées sur la figure. Avec ces valeurs, la formule précédente donne un indice spectral de
$-1,7$, en bon accord avec l'indice mesuré après le passage de l'onde de choc [19].

4.2 MÉCANISME DE DÉRIVE DANS LE GRADIENT DE CHAMP ASSOCIÉ AU FRONT D'ONDE. - Pour certains événements, la durée de l'accroissement d'intensité ne dépasse pas quelques minutes. On ne peut pas rendre compte de tels événements par un processus diffusif, comme celui que nous venons de décrire. Mais on remarque que lorsque surviennent de tels événements brefs («Shock Spike Events»), l'onde de choc est quasi perpendiculaire (la normale au front d'onde est sensiblement perpendiculaire au champ magnétique, autrement dit, le front d'onde et le champ magnétique sont quasi parallèles).

On attribue dans ce cas l'accélération des particules au mécanisme de dérive dans le gradient de champ présent au voisinage de l'onde de choc : les centres-guides des particules dérivent parallèlement au front d'onde, donc le long des lignes de force du champ électrique $\mathbf{E}=-\frac{1}{c} \mathbf{v} \wedge \mathbf{B}$ induit par le mouvement du plasma magnétisé. Par suite, ces particules gagnent de l'énergie (ici $v$ est la vitesse du plasma en aval du front d'onde dans un référentiel lié à l'onde de choc) (Fig. 9). 


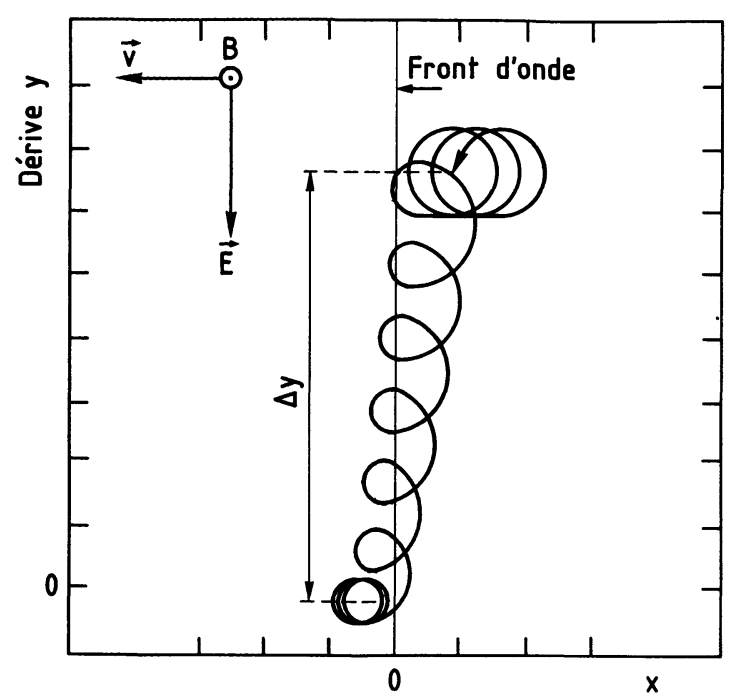

Fig. 9. - Accélération d'une particule par une onde de choc quasi perpendiculaire [21]. Mouvement d'une particule traversant l'onde de choc dans le référentiel du front d'onde. Une particule de charge $q$ gagne une énergie cinétique $\Delta T=q|\mathbf{E}| \Delta Y$.

[Acceleration of a particle by a quasi-perpendicular shock wave [21]. Trajectory of a particle which crosses the shock front, in the shock front frame of reference. A particle of charge $q$ gets an energy gain $\Delta T=q|\mathbf{E}| \Delta Y$.]

Pour des ondes de choc typiques se propageant dans l'espace interplanétaire, on observe une vitesse de plasma de l'ordre de 2 à 3 fois la vitesse d'Alfven dans le milieu, et une intensité hydrodynamique de l'onde de choc $\mathcal{H}$ de 1,5 à 3 (rapport des densités de plasma en amont et en aval de l'onde de choc). L'intensité du champ magnétique interplanétaire est très variable et comprise entre 2 à 50 nanotesla environ ; pour une onde de choc perpendiculaire, le rapport des intensités du champ en amont et en aval du front d'onde est sensiblement égal à $\mathcal{H}$ [20]. L'accélération des particules peut être rapide quelques dizaines de gyropériodes - et importante : l'énergie finale de la particule est de l'ordre de 10 fois l'énergie initiale [21, 22, 23, 24].

Ainsi, les deux mécanismes d'accélération (Fermi et dérive dans le choc) se distinguent par l'orientation de la normale au front d'onde par rapport au champ magnétique (quasi parallèle ou oblique pour les premiers, quasi perpendiculaire pour les seconds), par la durée de l'événement (quelques heures pour les premiers, quelques minutes pour les seconds) et également par l'anisotropie des particules accélérées (faible anisotropie pour les premiers, comme on peut l'attendre pour un mécanisme diffusif, forte pour les seconds, avec, en aval du choc, un courant de particules dans la direction opposée au front d'onde).

Mais en fait, tous les événements ne peuvent entrer dans des catégories aussi tranchées. Par exemple le $2^{\mathrm{c}}$ mécanisme, agissant en présence de centres diffuseurs peut donner lieu, lui aussi à une accélération stochastique, se traduisant par un événement de longue durée et de faible anisotropie [25].

\section{Origine des particules accélérées. Densités d'éner- gie.}

Les particules accélérées ont sensiblement la composition du vent solaire. Elles ont donc probablement pour origine la queue de la distribution suprathermique du vent solaire. C'est ce que semble également montrer leur spectre d'énergie, qui est en continuité avec celui du vent solaire (Fig. 10) [25, 31].

La densité d'énergie des particules accélérées dans quelques événements typiques, est de 3 à 4 fois supérieure à la densité d'énergie du champ magnétique et est une fraction notable (30 à $40 \%$ ) de la densité d'énergie du mouvement convectif du plasma dans le référentiel de l'onde de choc [17].

Les particules, emportant une part significative de la densité d'énergie contenue dans le plasma constituent une composante importante du milieu, qui affecte la structure même de l'onde de choc [26, 27].

On observe globalement des densités d'énergie comparables pour les particules accélérées par les deux types de mécanismes mentionnés précédemment. Le mécanisme de Fermi produit cependant en général des particules de moindre énergie, mais en plus grand nombre [28]. Ainsi, du point de vue de l'efficacité du mécanisme par particule, les ondes de choc quasi perpendiculaires apparaissent comme les accélérateurs les plus efficaces du milieu interplanétaire.

\section{Conclusion.}

Nous avons essayé de passer en revue les événements particulaires associés aux ondes de choc produites dans l'espace interplanétaire par interaction d'un vent solaire rapide avec un vent solaire lent. On observe dans l'héliosphère d'autres manifestations de l'association ondes de choc-particules accélérées : c'est en particulier le cas au voisinage des planètes possédant une magnétosphère, comme la terre. Le vent solaire arrivant sur cet obstacle avec une vitesse supersonique, une onde de choc («Bow Shock») est produite. Ici encore, les deux mécanismes d'accélération jouent un rôle : dans la partie de l'onde quasi perpendiculaire, on observe un faisceau de particules accélérées, de quelques dizaines de $\mathrm{keV}$, qui remontent le long des lignes de force du champ, et dans la partie où l'onde est quasi parallèle, on observe une population de particules diffuses [29].

Enfin, les rayons cosmiques solaires, émis par le soleil lors des éruptions chromosphériques («Solar Flare Particles ») et qui ont couramment des énergies de quelques centaines de MeV (et même exceptionnellement de quelques $\mathrm{GeV}$ ), apparaissent étroite- 

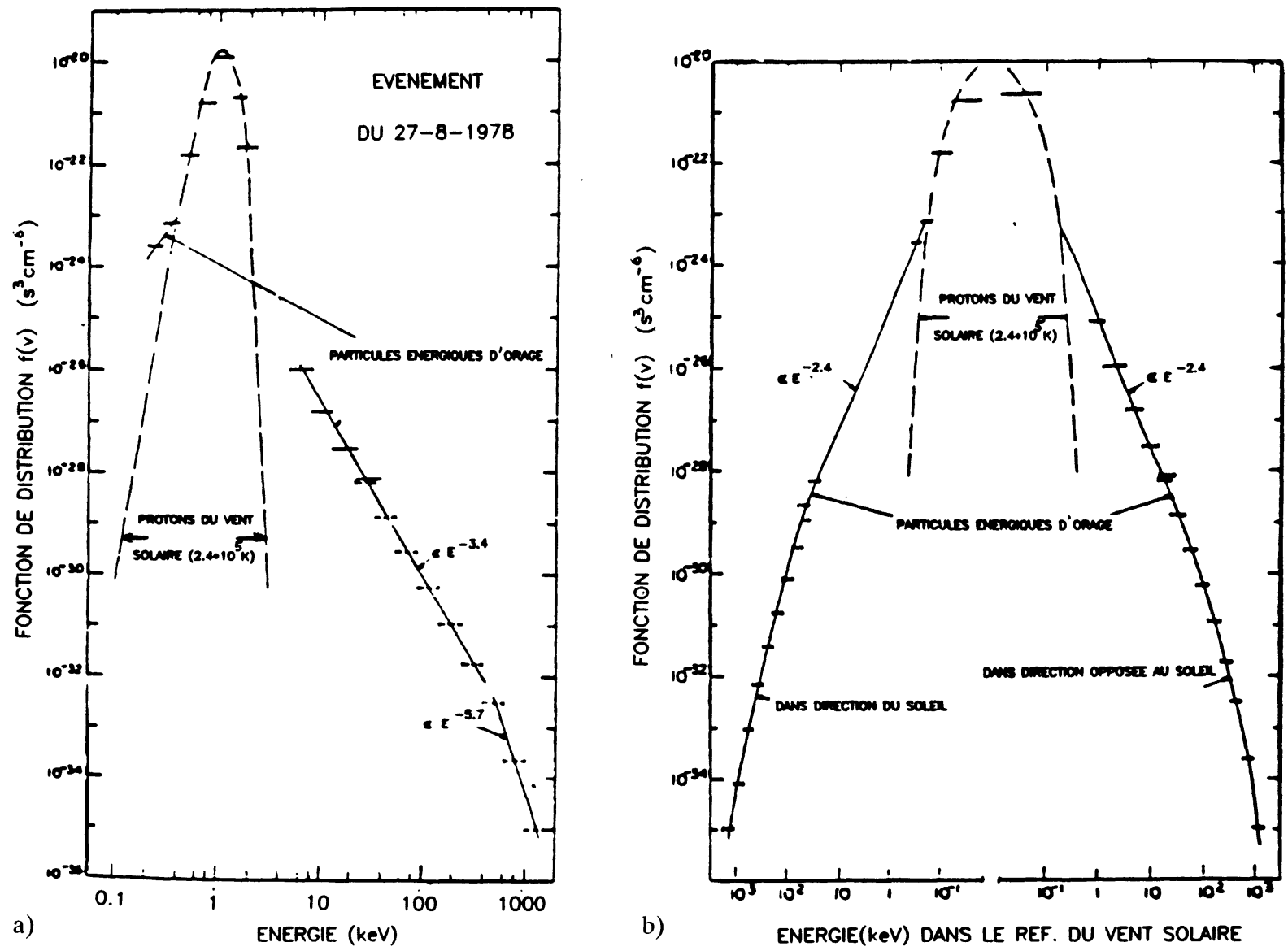

Fig. 10. - Fonction de distribution en vitesse des ions interplanétaires (vent solaire + particules accélérées) mesurée par le satellite ISEE 3 lors de l'événement particulaire d'orage du 27 août 1978 (d'après [31]). a) A gauche, dans le système de référence du satellite. b) A droite, dans un système de référence lié au vent solaire (après avoir retranché la composante de particules alpha du vent solaire). La courbe pointillée à travers les données est une gaussienne en vitesse, correspondant à une température des protons du vent solaire de $2,4 \times 10^{5} \mathrm{~K}$, une densité de protons de $25 \mathrm{~cm}^{-3}$, et centrée sur une vitesse de vent solaire de $445 \mathrm{~km} / \mathrm{s}$.

[Velocity distribution function of interplanetary ions (solar wind + accelerated ions) measured by the ISEE 3 satellite during the energetic storm particle event of 27 August 1978 (from [31]). a) On the left side, in the satellite frame of reference. b) On the right side, in the solar wind frame of reference. The dashed curve at the center is a gaussian in velocity corresponding to a solar wind proton temperature of $2.4 \times 10^{5} \mathrm{~K}$ and a proton density of $25 \mathrm{~cm}^{-3}$, centered on a solar wind velocity of $445 \mathrm{~km} / \mathrm{s}$.]

ment associés à des ondes de choc. Ces dernières peuvent être détectées depuis le sol pendant leur propagation dans la basse couronne grâce aux émissions radio qu'elles provoquent (émissions dites de type II [30].

Ainsi l'accélération des particules par ondes de choc est un phénomène d'une grande importance dans l'héliosphère. Les connaissances acquises dans ce domaine, grâce aux observations in situ effectuées par les satellites et sondes d'exploration interplanétaire, ont d'importantes implications astrophysiques.
On admet en effet généralement que les rayons cosmiques galactiques, particules d'énergie supérieure au $\mathrm{GeV}$, sont accélérés par les ondes de choc qui se propagent dans l'espace interstellaire, après avoir été engendrées par les supernovae.

\section{Remerciements.}

Je tiens à remercier J. P. Meyer, T. Montmerle et $M$. Tagger pour leurs pertinentes critiques et suggestions. 


\section{Bibliographie}

[1] Cesarsky, C. J. and Meyer, J. P.; Europhys. News 15 (1984) 2, 12.

[2] PARKeR, E. N., Interplanetary Dynamical Processes (New York, Interscience Publishers) 1963.

[3] Hundhausen, A. J., Coronal Expansion and Solar Wind (Springer Verlag ed.) 1972.

[4] TOPTYGIN, I. N., Cosmic Rays in Interplanetary magnetic Fields (D. Reidel Publishers) 1985.

[5] LeE, M. A., Rev. Geophys. and Space Phys. 21 (1983) 324.

[6] Decker, R. B., Pesses, M. E., Krimigis, S. M., J. Geophys. Res. 86 (1981) 8819.

[7] Pyle, R. R., Simpson, J. A., BaRnes, A. and MihaLOV, J. D., Astrophys. J. 282 (1984) L107.

[8] SARris, E. T. and Krimigis, S. M., Astrophys. J. 298 (1985) 676.

[9] McDonald, F. B., 17th Int. Cosmic Ray Conf., Paris 13 (1981) 199.

[10] SCHOler, M., 8th European Cosmic Ray Symposium, Rome, Iucci et al. Eds. (1983), p. 1.

[11] Barnes, C. W. and Simpson, J., Astrophys. J. 210 (1976) L91.

[12] SMITH, E. J., in « Collisionless Shocks in the Heliosphere », Geophys. monographs 35, American geophys. Union (1985) ; p. 69.

[13] Simpson, J. A., Smith, E. J. and Tsurutani, B., in The Sun and the Heliosphere in Three Dimensions, Marsden Ed. (D. Reidel) 1985, p. 319.
[14] LeE, M. A. and Fisk, L. A., Space Sci. Rev. 32 (1982) 205.

[15] LeE, M. A., J. Geophys. Res. 88 (1983) 6109.

[16] Decker, R. B., J. Geophys. Res. 88 (1983) 9959.

[17] Sanderson, T. R., Adv. Space Res. 4 (1984) 305.

[18] JoKIPII, J. R., Astrophys. J. 255 (1982) 716.

[19] SCHOler, M. et al., J. Geophys. R. 88 (1983) 1977.

[20] Wilkerson, T. D., Solar Phys. 6 (1969) 44.

[21] SARRIS, E. T., 8th European Cosmic Ray Symposium, Rome, Iucci et al. Eds. (1983) p. 17.

[22] JokIPII, J. R., Astrophys. J. 255 (1982) 716.

[23] Forman, M. A. and WebB, G. M., in Collisionless Shocks in the Heliosphere (1985), op. cit., p. 91.

[24] Decker, R. B. and Vlahos, L., J. Geophys. Res. 91 (1986) 13349.

[25] Gosling, J. T., Space Sci. Rev. 34 (1983) 113.

[26] Ellison, D. C., These, Catholic Univ., Washington (1981).

[27] Ellison, D. C., J. Geophys. Res. 90 (1985) 29.

[28] SARris, E. T. and Krimigis, S. M., Astrophys. J. 298 (1985) 676.

[29] Russel, C. T. and Hoppe, M. M., Space Sci. Rev. 34 (1983) 155

[30] Forman, M. A., Ramaty, R. and Zweibel, E. G., in Physics of the Sun, Peter Sturrock Ed. (D. Reidel) Vol. II (1986) p. 249.

[31] G.osling, J. T. et al., J. Geophys. Res. 86 (1981) 547.

[32] Drury, O., Cette Conf. 